\title{
Organic Computing - A Generic Approach to Controlled Self-organization in Adaptive Systems
}

\author{
Hartmut Schmeck \\ Institute AIFB, Karlsruhe Institute of Technology \\ Kaiserstr. 89, 76133 Karlsruhe, Germany \\ hartmut.schmeck@kit.edu
}

\begin{abstract}
Organic Computing is a recent paradigm for the design and management of complex technical systems, addressing the need for adaptive, selforganising systems which are capable of dealing with changing requirements and unanticipated situations in a robust and trustworthy way and which allow an external "observer" or "user" to interfere and influence the system whenever it does not show acceptable behaviour by itself. Research in this area has been supported by a priority program of the German Research Foundation!. The talk will present the generic observer/controller architecture of Organic Computing. An essential concept of this architecture is the combined use of online and offline learning. The application of these concepts will be described with respect to our research on organic traffic control and on smart energy systems.
\end{abstract}

${ }^{1}$ http: / /www . organic-computing.de/SPP 